\title{
ÉTUdE PAR LA CHROMATOGRAPHIE EN PHASE GAZEUSE \\ DE LA MATIÈRE GRASSE DU LAIT DE VACHES. VARIATIONS DE LA COMPOSITION EN ACIDES GRAS OBSERVÉES EN BELGIQUE
}

\author{
par
}

\author{
A.-L. GUYOT et E.-F. PIRAUX \\ Station laitière de l'Etat à Gembloux
}

La chromatographie en phase gaz-liquide nous a permis d'entreprendre une étude approfondie de la matière grasse des beurres belges. La première partie de cette étude, publiée en 1964, traitait avant tout des variations de composition en acides gras des beurres de laiteries [1]. Les observations s'étendaient sur une année du 1er mai 1963 au 30 avril 1964. Depuis cette date, nous avons continué nos observations, non seulement sur des beurres de laiteries, mais aussi sur de nombreux beurres de fermes et des laits individuels. Nous ferons part des résultats obtenus dans le présent travail.

\section{Procédé}

La transestérification en milieu alcalin des acides gras des matières grasses alimentaires nous ayant donné d'excellents résultats, sans sérieuses difficultés d'exécution, nous continuerons à utiliser ce procédé.

Les esters méthyliques en solution concentrée dans l'hexane, sont séparés, en chromatographie, sur une colonne polaire chauffée à une température proche de $200^{\circ} \mathrm{C}$.

\section{Modalités de techniques}

Appareil : Perkin Elmer 116 E.

Colonne : 1 colonne E.G.S. (éthylène glycol succinate) $2 \mathrm{~m}$.

Température de la colonne : $\pm 200^{\circ} \mathrm{C}$.

Gaz vecteur : hydrogène à une pression de 0,7 à 1,2 atm.

Débit : $\pm 60 \mathrm{ml} \mathrm{H}_{2} / \mathrm{min}$ à $21^{\circ} \mathrm{C}$.

Détecteur : Hot wire J. 6230 ma.

Enregistreur : Sargent 1 pouce/min.

Injection : 0,4 à 1,2 microlitre.

La colonne E.G.S. en acier inoxydable, d'une longueur de $2 \mathrm{~m}$, d'un diamètre intérieur de $4,65 \mathrm{~mm}$ contient comme support inerte de la célite (60-100 mesh) imprégnée de 15 p. 100 d'un polyester de succinate d'éthylène glycol. 


\section{Analyse quantitative}

Dans les conditions décrites ci-dessus, plus de 99 p. 100 des esters méthyliques des acides gras des beurres ont un temps de rétention inférieur à quinze minutes.

Les aires des pies sont calculées par triangulation, multipliées par le coefficient d'atténuation éventuel, et un facteur de correction établi par étalonnage.

Le rapport de chaque aire corrigée à l'aire totale x 100 , constitue le pourcentage en esters méthyliques de l'acide gras auquel le pic se rapporte.

L'étalonnage a été effectué à partir d'un mélange d'esters méthyliques purs du commerce, réalisé dans des proportions voisines de celles rencontrées dans les beurres [1]

Nous avons tenu compte des facteurs de correction suivants :

Butyrate de méthyle : 1

Caproate de méthyle : 0,84

Caprylate de méthyle : 0,88

Caprate de méthyle : 0,92

Laurate de méthyle : 0,92

Myristate de méthyle : 0,95

Palmitate de méthyle : 1,00

Stéarate de méthyle : 1,05

Oléate de méthyle : 1,09

Linoléate de méthyle : 1,12

Linolénate de méthyle : 1,16

\section{Variations mensuelles de la composition en acides gras de la matière grasse butyrique}

Les causes des variations de la composition en acides gras de la matière grasse butyrique sont multiples et très diverses. Les principales sont le régime alimentaire des vaches laitières, la période de lactation, la race, l'individu même. La cause primordiale toutefois, réside dans le régime alimentaire. Un régime alimentaire normal varie naturellement suivant les saisons. Au cours d'une année, il suffit donc de suivre régulièrement l'évolution de la composition en acides gras d'un beurre d'origine bien déterminée, pour étudier l'influence du régime alimentaire courant.

Deux ou trois fois par mois, au cours de 1964, les taux des divers acides gras d'un beurre de ferme de la région de Gembloux ont été relevés et la moyenne mensuelle a été calculée.

Les acides gras du beurre, à chaîne carbonée voisine, variant rarement de façon indépendante, nous avons également étudié les rapports moyens suivants : $\mathrm{C} 6 / \mathrm{C} 8, \mathrm{C} 12 / \mathrm{C} 10, \mathrm{Cl4} / \mathrm{C} 12, \mathrm{C} 16 / \mathrm{C} 14$, C18 1 :/C16, C18 $1: / \mathrm{C} 18$ et $\mathrm{C18} 3: / \mathrm{C} 182$ :

Les résultats de ces analyses sont repris dans le tableau 1. Ils ont donné lieu à la confection des graphiques en figures 1 à 15 . 


\section{TABLEAU - 1}

ÉVOLUTION MENSUELLE DE LA COMPOSITION EN ACIDES GRAS DE LA MATIk̀RE GRASSE BUTYRIQUE EN 1964 Beurres de la region de Gembloux

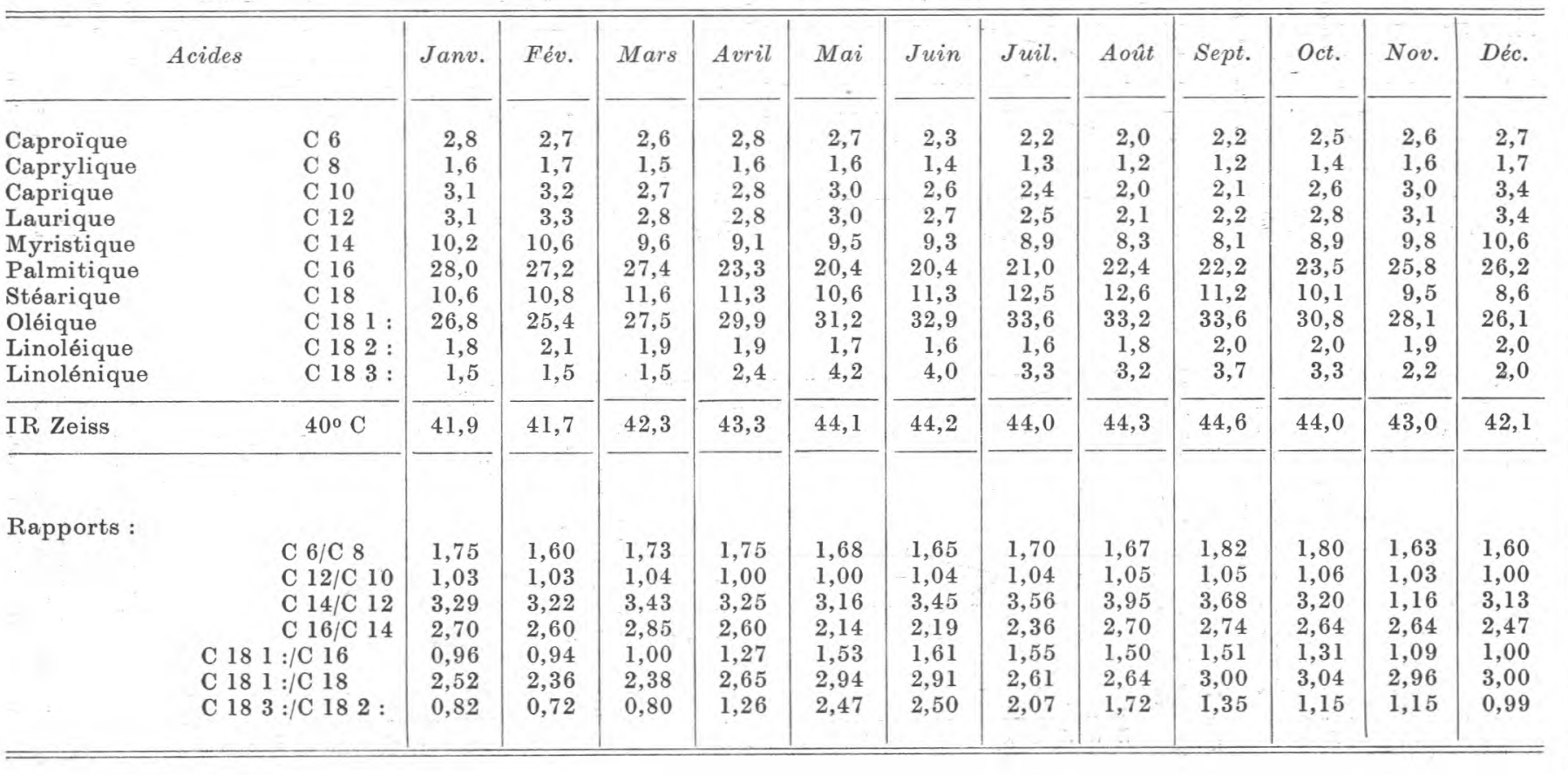




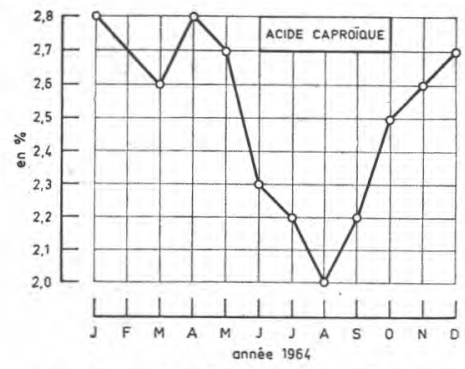

Fig. 1. - Variations pendant un an du taux d'acide caproïque de la graisse butyrique.

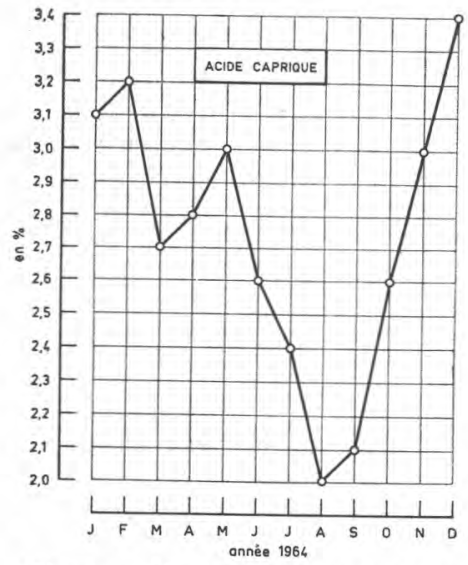

Fig. 3. - Variations pendant un an du taux d'acide caprique de la graisse butyrique.

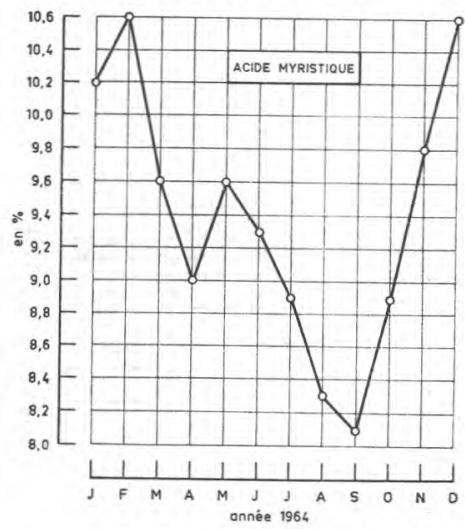

Fig. 5. - Variations pendant un an du taux d'acide myristique de la graisse butyrique.

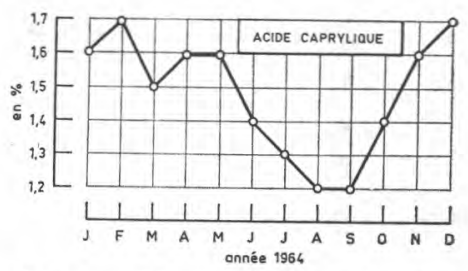

Fig. 2. - Variations pendant un an du taux d'acide caprylique de la graisse butyrique.

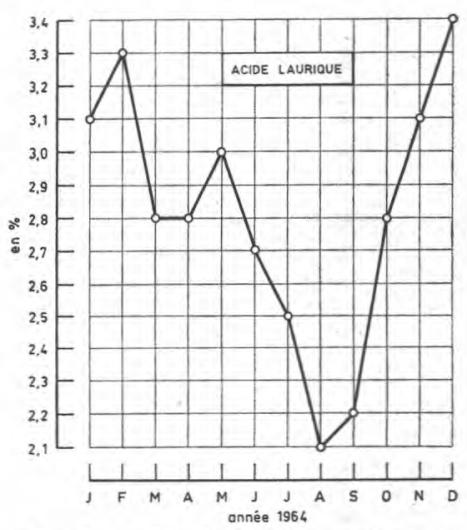

Fig. 4. - Variations pendant un an du taux d'acide laurique de la graisse butyrique.

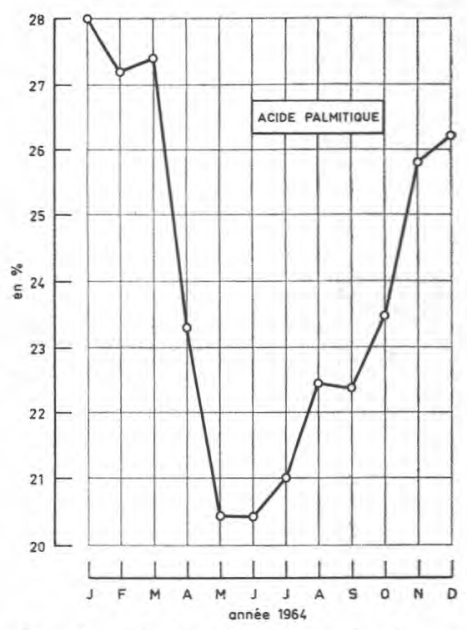

Fig. 6. - Variations pendant un an du taux d'acide palmitique de la graisse butyrique. 


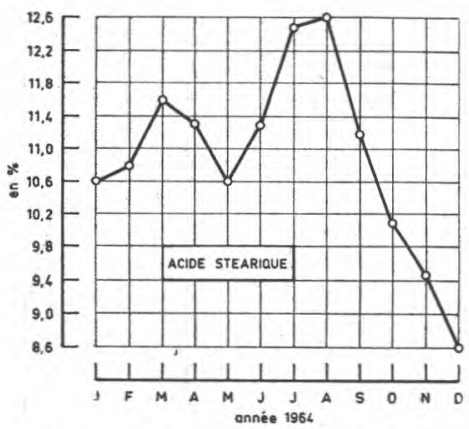

Fig. 7. - Variations pendant un an du taux d'acide stéarique de la graisse butyrique.

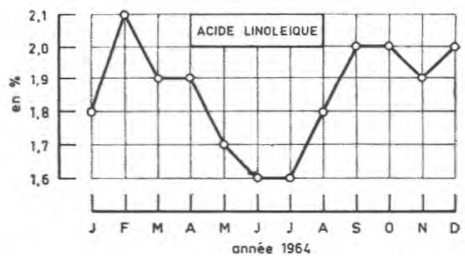

Fig. 9. - Variations pendant un an du taux 'd'acide linoléique de la graisse butyrique.

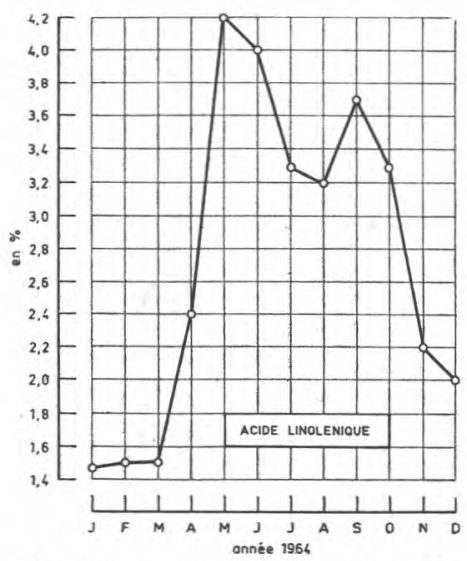

Fig. 10. - Variations pendant un an du taux d'acide linolénique de la graisse butyrique.

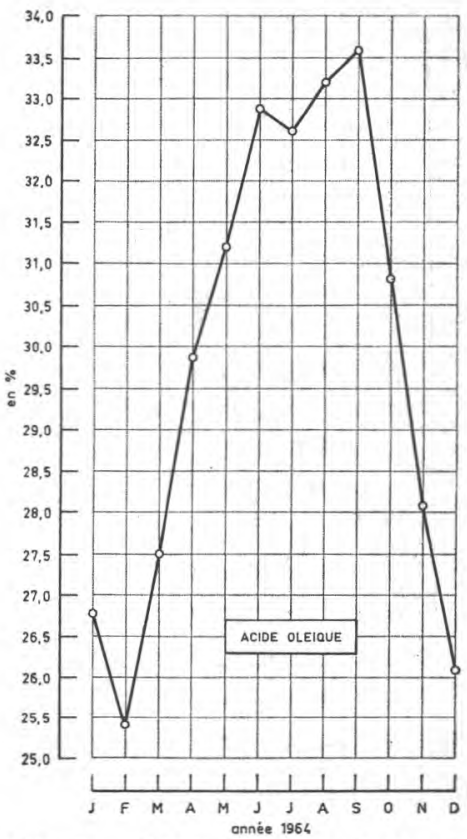

Fig. 8. - Variations pendant un un an du taux d'acide oléique de la graisse butyrique.

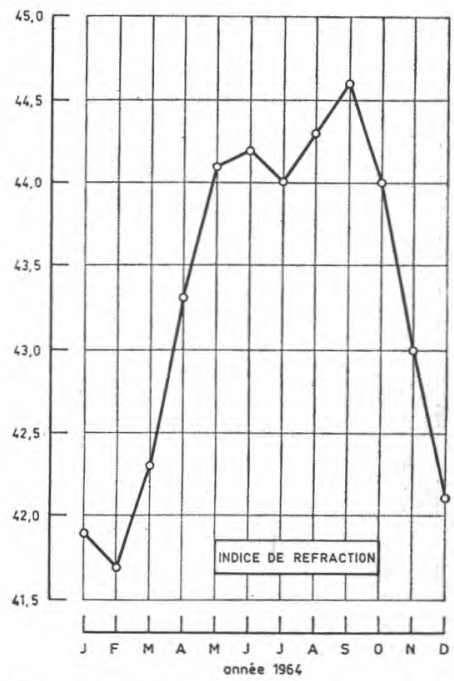

Fig. 11. - Variations pendant un an de l'indice de réfraction Zeiss à $40^{\circ} \mathrm{C}$ de la graisse butyrique. 


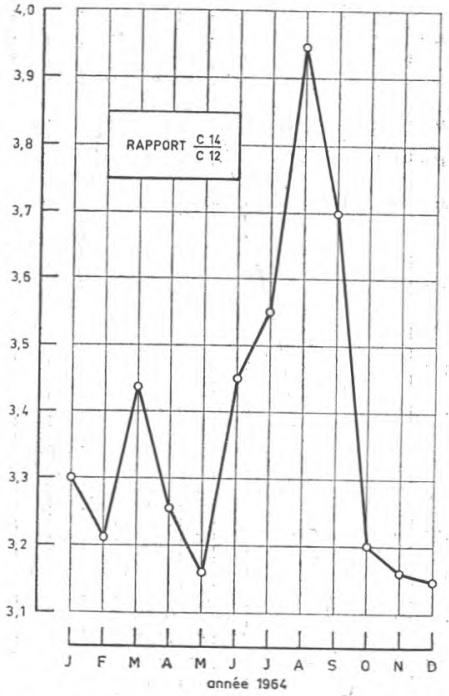

Fig. 12. - Variations pendant un an du rapport $\frac{\mathrm{C} 14}{\mathrm{C} 12}$

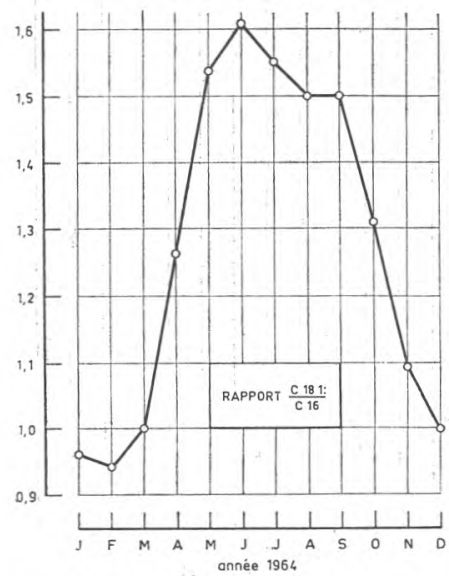

Fig. 14. - Variations pendant un an du rapport $\frac{\mathrm{C} 181 \text { : }}{\mathrm{C} 16}$

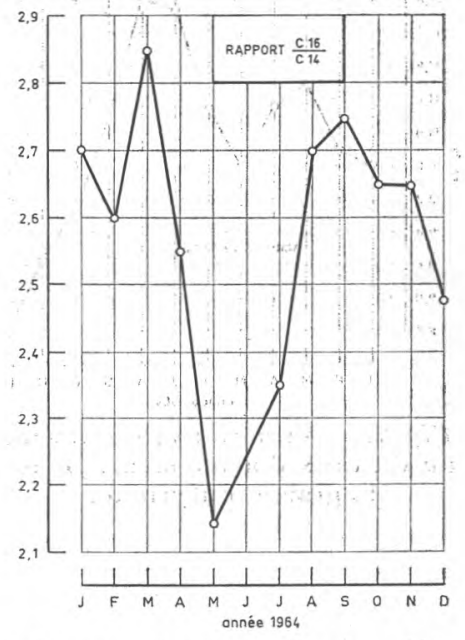

Fig. 13. - Variations pendant un an du rapport $\frac{\mathrm{C} 16}{\mathrm{C} 14}$

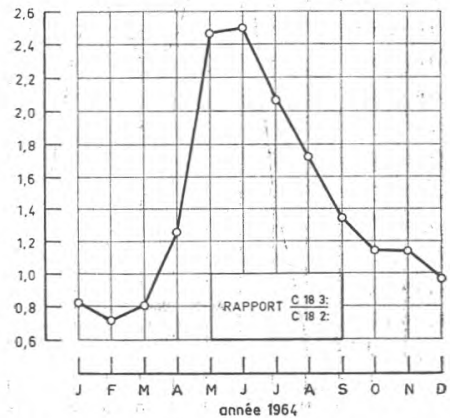

Fig. 15. - Variations pendant un an du rapport $\frac{\mathrm{C} 183 \text { : }}{\mathrm{C} 182 \text { : }}$ 


\section{A. Variations du taux des acides gras.}

\section{a) Les acides inférieurs.}

Les taux des acides caproïque, caprylique, caprique, laurique et myristique sont à leur valeur maximale en plein hiver. Ils décroissent quelque peu en mars, augmentent légèrement en avril et mai, au moment de la mise en pâture pour redescendre régulièrement vers une valeur minimale en été. Dès le mois de septembre, les taux des acides inférieurs s'élèvent tout aussi régulièrement vers les valeurs maximales d'hiver.

En raison de l'imprécision de la méthode chromatographique relative au dosage de l'acide butyrique, il nous est impossible de donner par graphique les variations au cours d'une année de la teneur en acide butyrique des beurres. Cependant, toutes nos observations semblent indiquer que le taux d'acide butyrique varie dans le même sens que le taux des autres acides inférieurs.

b) L'acide palmitique.

Le taux d'acide palmitique est maximum en hiver, il subit une chute brutale dès la mise en pâture en avril ou mai, augmente à nouveau en juillet, passe par un court palier en août et septembre pour s'élever très fort dès le mois d'octobre.

c) L'acide oléique.

Le taux d'acide oléique varie approximativement en sens inverse par rapport au taux d'acide palmitique. Minimum en février, il s'élève régulièrement jusqu'en mai, se stabilise au moment où les pâtures restent vertes, croît légèrement jusqu'en août ou septembre. La chute est alors brutale et régulière jusqu'en décembre, plus lente jusqu'en février.

Il est significatif que la courbe de variations du taux d'acide oléique et la courbe de variations de l'indice de réfraction mesuré au butyromètre Zeiss à $40^{\circ} \mathrm{C}$, ont une allure fort semblable.

d) L'acide stéarique.

L'acide stéarique semble varier de façon parallèle à l'acide oléique. Les teneurs maximales se rencontrent en été et les teneurs minimales en hiver.

\section{e) L'acide linolénique.}

La courbe des variations du taux d'acide linolénique a sensiblement la même allure que la courbe des variations du taux d'acide oléique. Il existe deux valeurs maximales en mai ou juin, au moment de la pousse des herbages, et en août-septembre. 


\section{f) L'acide linoléique.}

Contrairement aux autres acides à 18 atomes de carbone, le taux d'acide linoléique est sensiblement moins éleyé en été qu’en hiver.

\section{B. Variations de quelques rapports caractéristiques.}

a) Le rapport $C 14 / C 12$.

Le rapport $\mathrm{C14} / \mathrm{C} 12$ est très bas en hiver et maximum à la fin de l'été. Au moment de la mise en pâtures, ce rapport díminue très fort jusqu'à des valeurs souvent inférieures aux chiffres observés en hiver.

b) Le rapport $C 16 / C 14$.

La courbe de variations du rapport $\mathrm{Cl6} / \mathrm{Cl} 4$ montre un pic très net vers le bas en mai. L'effet maximum de la mise en pâture est marqué par cette courbe.

c) Les rapports $C 181: / C 16$ et $C 183: / C 182$ :

Les courbes de variations de ces deux rapports suivent en l'accentuant l'allure des courbes de variations des taux des acides oléique et linolénique ou de l'indice de réfraction mesuré au butyroréfractomètre Zeiss à $40^{\circ} \mathrm{C}$.

d) Les rapports $C 6 / C 8$ et $C 12 / C 10$.

Ces rapports sont relativement indépendants de la période de l'année. Toutefois, le rapport $\mathrm{Cl} / \mathrm{Cl0}$, varie en fonction du régime alimentaire. Il augmente considérablement lors de l'adjonction dans le régime alimentaire des vaches laitières, de suppléments comprenant des tourteaux de coprah ou de palmiste. Comme ces suppléments sont généralement donnés en hiver ou au cœur d'un été très sec, lorsque les pâtures sont très maigres, le rapport $\mathrm{C} 12 / \mathrm{Cl0}$, du moins dans certaines régions, est plus élevé pendant ces périodes de l'année [2].

\section{Variations des courbes}

Les courbes reproduites dans ce travail sont spécifiques d'un seul beurre, d'origine bien déterminée, dont les analyses s'échelonnent sur une année. Pendant près de trois ans, nous avons étudié de nombreux autres échantillons et établi un bon nombre de graphiques.

D'une année à l'autre ou d'une région à l'autre, les courbes peuvent subir de légères variations en fonction des saisons ou du régime alimentaire. Cependant, au moins pour des régions voisines, 
l'allure reste la même. Le léger décalage des saisons, suivant les années ou suivant les régions, a pour effet un décalage correspondant des courbes dans le sens horizontal.

\section{Conclusions}

Si les taux d'acides gras ou leurs rapports sont éminemment variables, on ne peut leur attribuer des valeurs extrêmes pendant n'importe quel mois de l'année ou pour n'importe quel régime alimentaire des vaches laitières.

Les taux les plus élevés en acides inférieurs et en acide palmitique se rencontrent en hiver, et inversement les taux les plus élevés en acides supérieurs se rencontrent en été.

Le taux d'acide linoléique est assez constant.

La période de mise en pâtures est caractérisée par les chiffres les moins élevés pour le taux d'acide palmitique, le rapport $\mathrm{C} 16 / \mathrm{Cl} 4$ et le rapport $\mathrm{Cl} / \mathrm{C12}$.

De l'adjonction de tourteaux de coco ou de palmiste, dans le régime alimentaire du bétail découlera des teneurs en acide laurique et en acide myristique plus grandes, le rapport $\mathrm{C} 12 / \mathrm{Cl0}$ le plus élevé et le rapport $\mathrm{C14/ \textrm {Cl } 2}$ le plus faible.

Connaissant la composition en acides gras d'un beurre, on peut donc tirer de nombreux renseignements quant à son origine, la période à laquelle il a été fabriqué ou, éventuellement, le régime alimentaire des vaches laitières.

$\mathrm{Au}$ moins par région, il est possible de distinguer assez facilement les beurres d'été des beurres d'hiver et fréquemment, les beurres de mai ou juin des autres beurres d'été. On pourra également observer les limites des variations de certains taux d'acides gras ou de certains rapports pour des beurres d'été ou des beurres d'hiver [1].

Ces observations seront d'un intérêt évident sur le plan de la recherche des falsifications par addition de graisses étrangères au beurre [1], [2], [3].

\section{Variations extrêmes de la composition en acides gras de la matière grasse butyrique observées en 1963, 1964, 1965 sur des échantillons de beurres ou de laits belges}

Nous présentons en figures 16 à 21, quelques chromatogrammes établis à partir de beurres ou de laits de ferme. Les échantillons ont été choisis parmi les cas observés de variations extrêmes de la matière grasse butyrique du lait de vaches. Ils sont significatifs : 

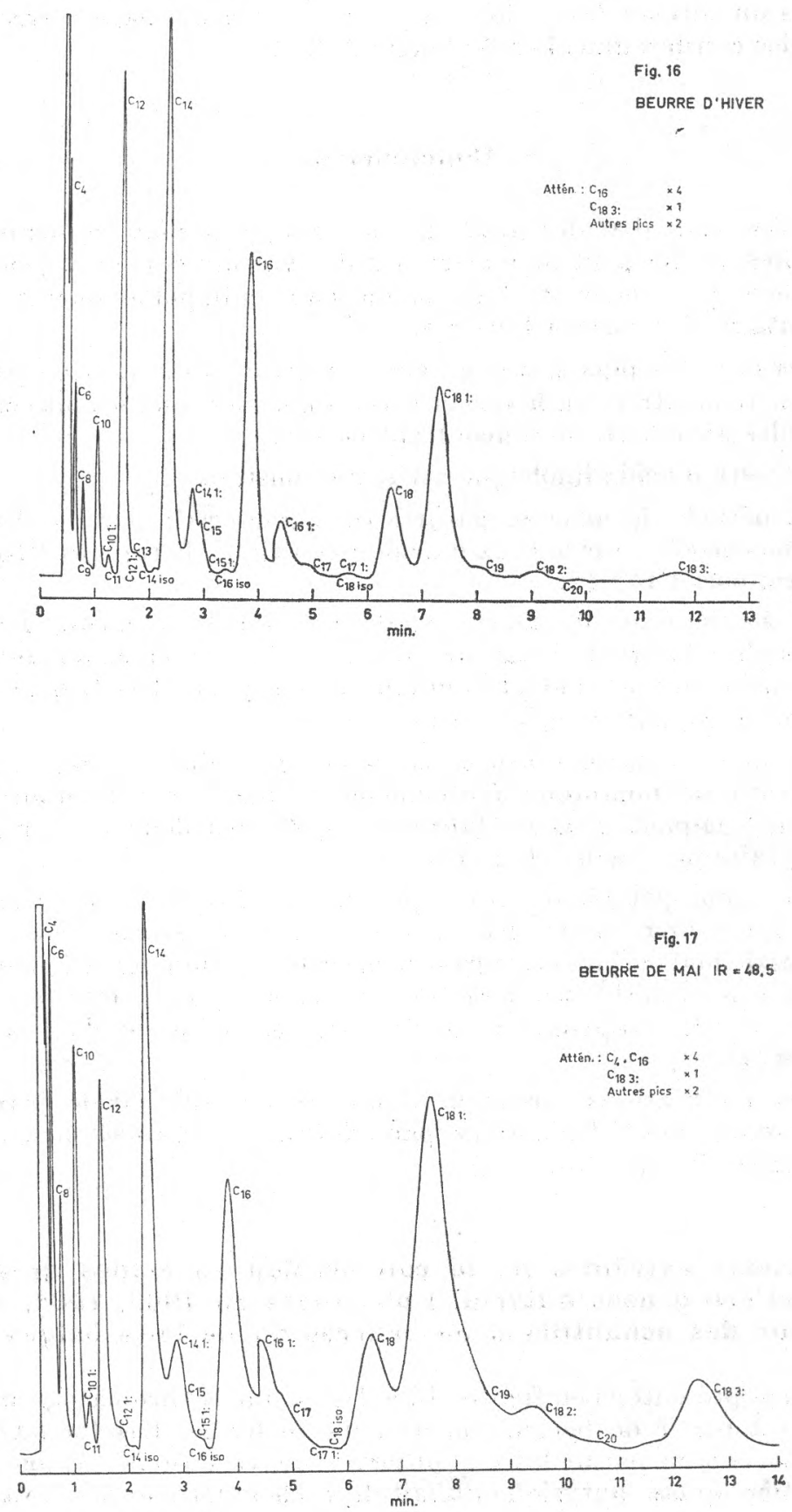

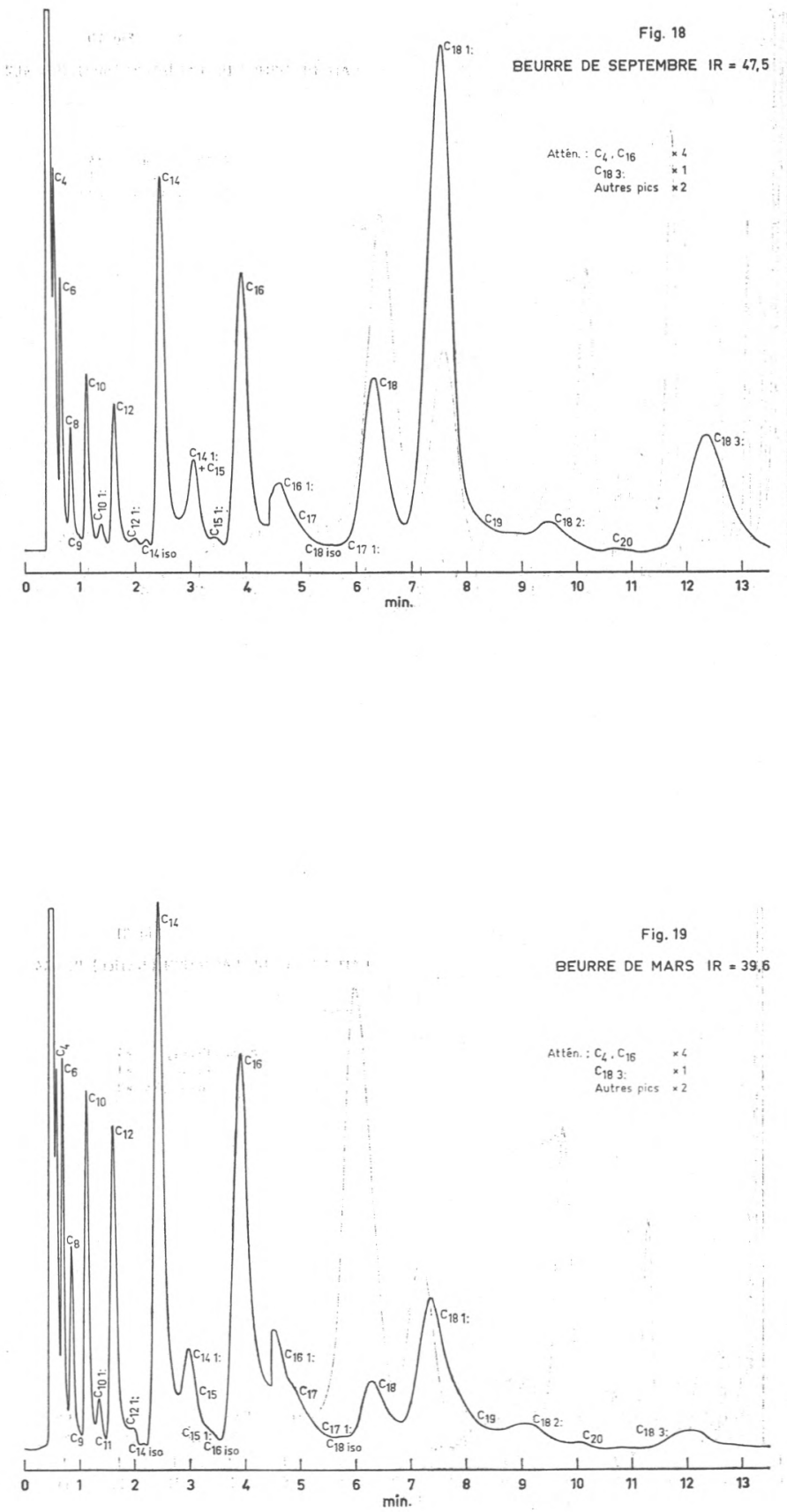

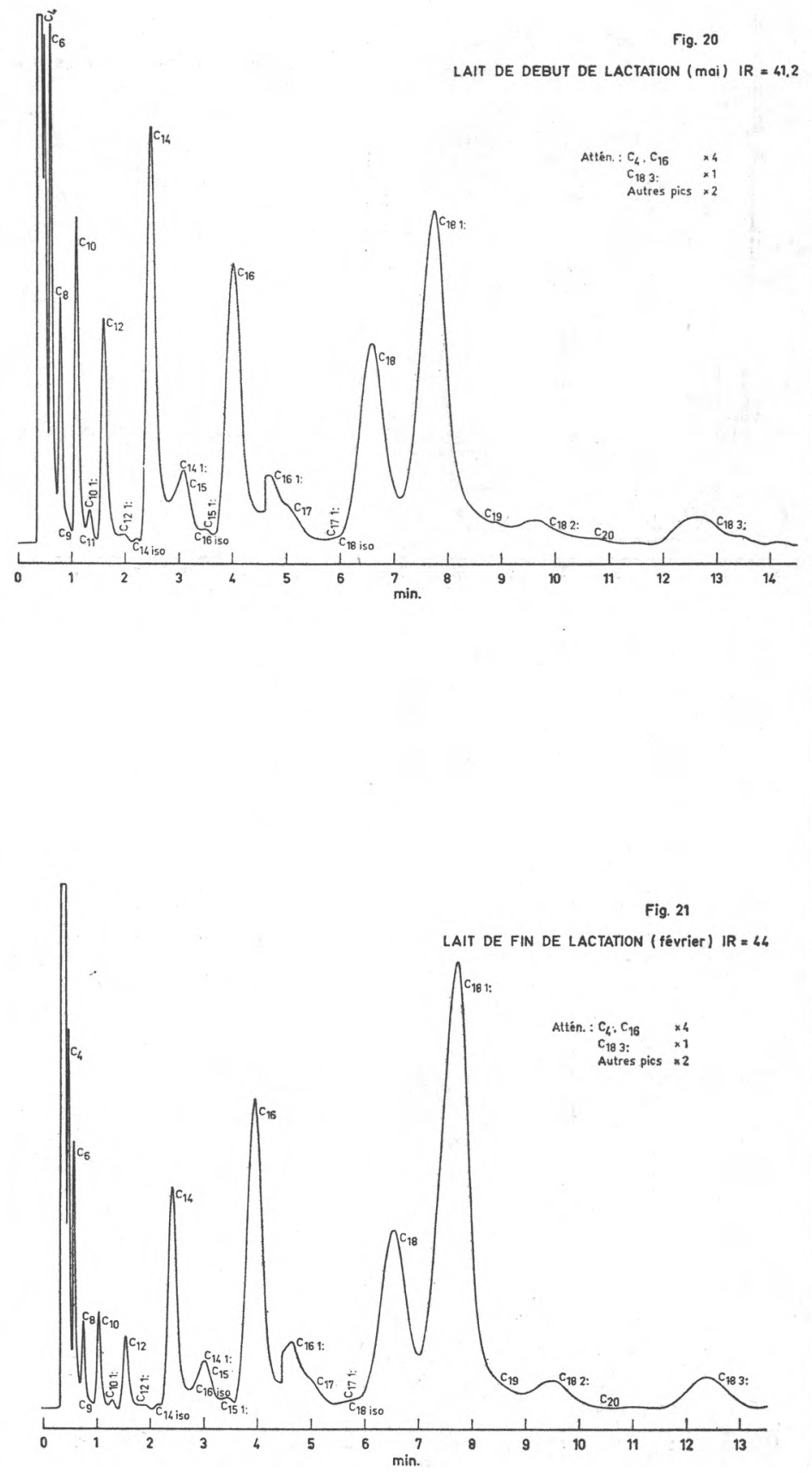
a) D'un régime alimentaire spécial des vaches laitières.

Le chromatogramme en figure 16, est réalisé à partir d'un beurre de ferme d'hiver fabriqué avec la crème du lait de vaches recevant un régime alimentaire très riche en tourteaux de coco.

b) D'un régime alimentaire normal, variant suivant les saisons.

En figure 17, est représenté le chromatogramme d'un beurre de ferme de mai, au moment où les vaches trouvent exclusivement leur nourriture dans les pâtures nouvellement poussées.

En figure 18, il s'agit d'un chromatogramme de beurre de ferme du début du mois de septembre.

En figure 19, le chromatogramme est celui d'un beurre de ferme du début du mois de mars.

c) De la période de lactation.

Le chromatogramme en figure 20 a été établi à partir de la matière grasse d'un lait individuel de mai, venant d'une vache fraîchement vêlée, tandis que le chromatogramme en figure 21 est celui d'un lait individuel de février, venant d'une vache en fin de période de lactation.

TABLEAU 2

ANALYSE DES ÉCHANTILLONS DONT LES CHROMATOGRAMMES SONT PRÉSENTÉS EN FIGURES 16 A 21 (P. CENT ESTERS MÉthyLIQUES DES ACIDES GRAS)

\begin{tabular}{|c|c|c|c|c|c|c|c|}
\hline \multicolumn{2}{|c|}{ Acides } & \multirow{2}{*}{$\begin{array}{c}\text { Fig. } 16 \\
3,3\end{array}$} & \multirow{2}{*}{$\begin{array}{c}\text { Fig. } 17 \\
3,8\end{array}$} & \multirow{2}{*}{$\begin{array}{c}\text { Fig. } 18 \\
3,4\end{array}$} & \multirow{2}{*}{$\begin{array}{c}\text { Fig. } 19 \\
4,1\end{array}$} & \multirow{2}{*}{$\begin{array}{c}\text { Fig. } 20 \\
5,0\end{array}$} & \multirow{2}{*}{$\begin{array}{r}\text { Fig. } 21 \\
3,3\end{array}$} \\
\hline Butyrique & $\mathrm{C} 4 \ldots \ldots \ldots \ldots$ & & & & & & \\
\hline Caproïque & $\mathrm{C} 6 \ldots \ldots \ldots \ldots$ & 2,4 & 2,8 & 2,2 & 3,0 & 3,5 & 1,8 \\
\hline Caprylique & $\mathrm{C} 8 \ldots \ldots \ldots$ & 1,2 & 1,7 & 1,2 & 1,8 & 2,1 & 0,8 \\
\hline Caprique & $\mathrm{C} 10 \ldots \ldots \ldots$ & 2,55 & 3,6 & 2,2 & 4,4 & 3,5 & 1,2 \\
\hline Caproléique & C 101: $(+\mathrm{C} 11)$ & 0,4 & 0,5 & 0,3 & 0,6 & 0,45 & 0,2 \\
\hline \multirow[t]{3}{*}{ Laurique } & $\mathrm{C} 12 \ldots \ldots \ldots \ldots$ & 10,7 & 4,2 & 2,3 & 5,0 & 3,3 & 1,2 \\
\hline & C 121: (+ C 13) & 0,7 & 0,3 & 0,1 & 0,3 & 0,2 & 0,1 \\
\hline & $\mathrm{C} 14$ iso ........ & 0,1 & 0,1 & 0,1 & 0,1 & 0,1 & $0, \hat{1}$ \\
\hline Myristique & C $14 \ldots \ldots \ldots \ldots$ & 16,6 & 11,0 & 8,4 & 14,4 & 9,8 & $5 ; 7$ \\
\hline \multirow[t]{2}{*}{ Myristoléique } & C 141: (+ C 15) & 4,1 & 2,9 & 2,9 & 3,7 & 2,7 & 1,9 \\
\hline & $\mathrm{C} 151: \mathrm{C} 16$ iso. & 0,1 & 0,2 & 0,1 & 0,2 & 0,2 & 0,1 \\
\hline Palmitique & C $16 \ldots \ldots \ldots$ & 28,2 & 21,1 & 20,1 & 35,8 & 22,7 & 26,7 \\
\hline Palmitoléique & C $161: \ldots \ldots \ldots$ & 3,9 & 3,5 & 3,1 & 4,3 & 3,4 & 2,9 \\
\hline \multirow[t]{2}{*}{ Margarique } & $\mathrm{C} 17 \ldots \ldots \ldots \ldots$ & 0,9 & 0,8 & 0,8 & 1,0 & 0,8 & 0,7 \\
\hline & $\mathrm{C} 171: \mathrm{C} 18$ iso. & 0,2 & 0,2 & 0,2 & 0,2 & 0,2 & 0,2 \\
\hline Stéarique & $\mathrm{C} 18 \ldots \ldots \ldots$ & 6,0 & 9,1 & 10,4 & 4,7 & 13,6 & 13,6 \\
\hline Oléique & C $181: \ldots \ldots \ldots$ & 16,0 & 28,3 & 34,2 & 13,0 & 24,6 & 35,2 \\
\hline Linoléique & C $182: \ldots \ldots \ldots$ & 1,2 & 1,7 & 1,7 & 1,6 & 1,4 & 1,9 \\
\hline \multirow[t]{2}{*}{ Linolénique } & C $183: \ldots \ldots \ldots$ & 0,9 & 3,4 & 6,1 & 1,1 & 1,7 & 1,8 \\
\hline & C $19 \ldots \ldots \ldots$ & 0,2 & 0,2 & 0,1 & 0,2 & 0,3 & 0,3 \\
\hline Arachidique & $\mathrm{C} 20 \ldots \ldots \ldots \ldots$ & 0,3 & 0,3 & 0,2 & 0,3 & 0,3 & 0,2 \\
\hline
\end{tabular}


Les résultats de l'analyse par chromatographie gazeuse des échantillons représentés par les chromatogrammes en figures 16 à 21 sont repris dans les tableaux 2 et 3 .

\section{TABLEAU 3}

ANALYSE DES ÉCHANTILLONS DONT LES CHROMATOGRAMMES SONT. PRÉSENTÉS EN FIGURES 16 A 21

\begin{tabular}{l|c|c|c|c|c|c}
\hline \hline $\begin{array}{c}\text { Rapports } \\
\text { caractéristiques }\end{array}$ & Fig. 16 & Fig. 17 & Fig. 18 & Fig. 19 & Fig. 20 & Fig. 21 \\
\cline { 2 - 6 } C 6/C 8 & 2,0 & 1,65 & 1,81 & 1,67 & 1,67 & 2,2 \\
C 12/C 10 & 4,2 & 1,17 & 1,04 & 1,13 & 0,94 & 1,01 \\
C 14/C 12 & 1,55 & 2,61 & 3,65 & 2,88 & 2,97 & 4,75 \\
C 16/C 14 & 1,70 & 1,92 & 2,39 & 2,49 & 2,32 & 4,68 \\
C 18 1 :/C 16 & 0,57 & 1,34 & 1,70 & 0,36 & 1,08 & 1,32 \\
C 18 1 :/C 18 & 2,66 & 3,11 & 3,29 & 2,77 & 1,80 & 2,59 \\
C 18 3:/C 18 2: & 0,75 & 2,00 & 3,60 & 0,70 & 1,22 & 0,95 \\
IR Zeiss 40 C & 40,0 & 43,5 & 47,5 & 39,6 & 41,2 & 44,0 \\
\end{tabular}

\section{Examen des résultats}

Figure 16.

Comme valeurs remarquables, nous retiendrons ici :

$$
\begin{aligned}
& \mathrm{C} 12=10,7 \% ; \mathrm{Cl}=16,6 \% ; \mathrm{Cl} 2 / \mathrm{Cl}=4,20 ; \mathrm{Cl} / 12=1,55 \\
& \text { et } \mathrm{C} 16 / \mathrm{Cl}=1,7 \text {. }
\end{aligned}
$$

Pour un régime alimentaire démuni de tourteaux de coprah ou de palmiste, les valeurs limites correspondantes que nous avons observées sont :

$$
\begin{aligned}
& \mathrm{C} 12= \pm 5 \% ; \mathrm{Cl}=14,4 \% ; \mathrm{C} 12 / \mathrm{Cl} 0=1,19 ; \mathrm{Cl} / \mathrm{C} 12=2,61 \\
& \text { et } \mathrm{C} 16 / \mathrm{C} 14=1,90 \text {. }
\end{aligned}
$$

Le rapport $\mathrm{C} 12 / \mathrm{C} 10=4,20$ est supérieur à celui observé par S. Kuzdzal et C. Paquot [4], qui avaient nourri pendant trois semaines un troupeau expérimental avec de l'herbe et un supplément constitué par un tourteau de coprah riche en huile. Du début à la fin de ce régime, le rapport $\mathrm{C} 12 / \mathrm{C} 10$ était passé de 1,06 à 3,60 .

\section{Figure 17.}

Le rapport $\mathrm{C} 14 / \mathrm{Cl} 2=2,61$ est à noter. Au moment de la mise en pâtures des vaches laitières, le rapport C14/C12 est le plus bas, souvent inférieur à 3 . En hiver, il est parfois compris entre 2,80 et 3. Pendant le reste de l'année, il semble toujours supérieur à 3 , sauf bien entendu, si le régime alimentaire des vaches contient une notable proportion de tourteaux de coprah ou de palmiste. 
La période de mise en pâtures est également celle où en moyenne, le taux d'acide palmitique est le plus faible.

Figure 18.

Remarquons ici le taux d'acide linolénique $=6,1 \%$, le rapport C18 $1: / \mathrm{Cl} 6=1,70$ et le rapport C18 $3: / \mathrm{Cl} 82:=3,60$. En août et en septembre, ces valeurs semblent les plus élevées.

Par contre, les taux des acides inférieurs sont les plus faibles.

\section{Figure 19.}

En fin d'hiver, on rencontre les taux les plus élevés des acides inférieurs et de l'acide palmitique $(\mathrm{Cl0}=4,4 \% ; \mathrm{Cl0} 1:=0,6 \%$; $\mathrm{Cl2}=5 \% ; \mathrm{Cl6}= \pm 36 \%$ pour un régime alimentaire démuni de tourteaux de coprah ou de palmiste).

Par contre, les taux des acides stéarique $(4,7 \%)$, oléique $(13 \%)$ et linolénique $(1,1 \%)$ de même que les rapports C18 1 :/C16 et C18 3 :/C18 2 : sont les plus faibles.

Figure 20.

Souvent, en début de période de lactation, le taux d'acide stéarique est important et le rapport C18 1 :/C18 est faible (minimum observé $=1,8$ ).

\section{Figure 21.}

En fin de période de lactation, le taux des acides inférieurs est plus faible, tandis que les rapports $\mathrm{Cl4} / \mathrm{Cl} 2$ et $\mathrm{Cl6} / \mathrm{Cl} 4$ sont particulièrement élevés.

\section{Teneurs en acides gras et rapports extrêmes observés en Belgique sur la matière grasse butyrique du lait de vaches}

Les teneurs en acides gras et rapports extrêmes consignés dans les tableaux 4 et 5 concernent la matière grasse d'échantillons de laits ou de beurres de fermes prélevés en Belgique, au cours des années 1963, 1964, 1965. Les valeurs observées recouvrent évidemment les valeurs correspondantes établies pour la matière grasse de beurres de laiteries belges [1].

\section{La composition en acides gras de la matière grasse des beurres et la recherche des falsifications}

Les valeurs reprises dans les tableaux 4 et 5 permettent de se rendre compte de l'étendue des variations de la composition de la matière grasse butyrique en fonction de certains facteurs. Ces 
valeurs ne sont pourtant pas limitatives, tant les éléments qui les font varier sont multiples et agissent différemment. Au moins, en Belgique, elles peuvent servir de base pour la recherche des falsifications par addition au beurre de matières grasses étrangères. L'étendue des variations de la composition en acides gras de la matière grasse butyrique démontre cependant la complexité du problème.

\section{TABLEAU 4}

VALEURS MINIMALES ET MAXIMALES DE LA TENEUR EN ACIDES GRAS (ESTERS MÉTHYLIQUES)

OBSERVÉES EN BELGIQUE AU COURS DES ANNÉES 1963, 1964, 1965

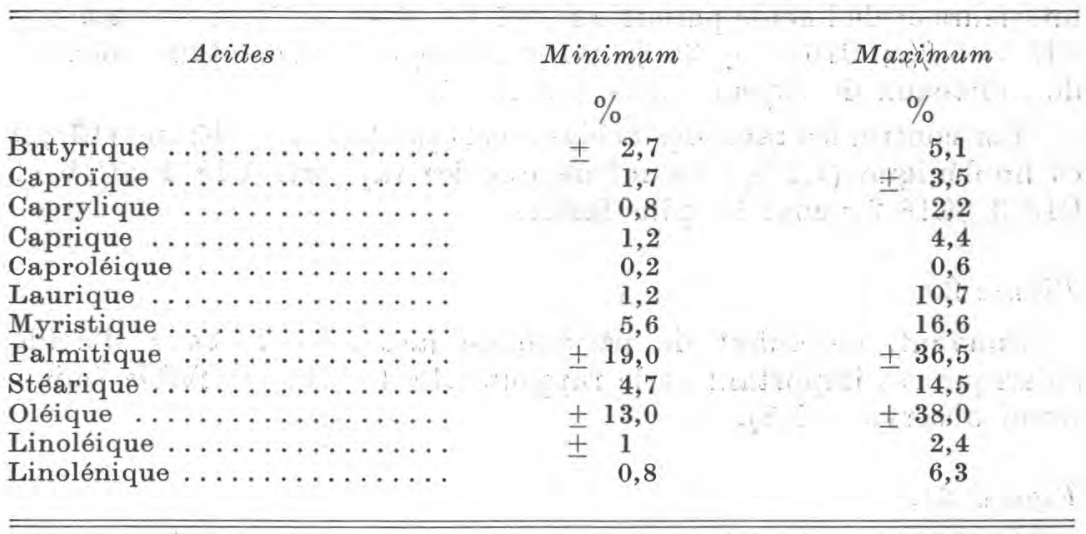

\section{TABLEAU 5}

VALEURS MINIMALES ET MAXIMALES DE CERTAINS RAPPORTS DE TENEURS EN ACIDES GRAS (ESTERS MÉTHYLIQUES) OBSERVÉES SUR LA MATIÈRE GRASSE BUTYRIQUE D'ORIGINE BELGE AU COURS DES ANNÉE 1963, 1964, 1965

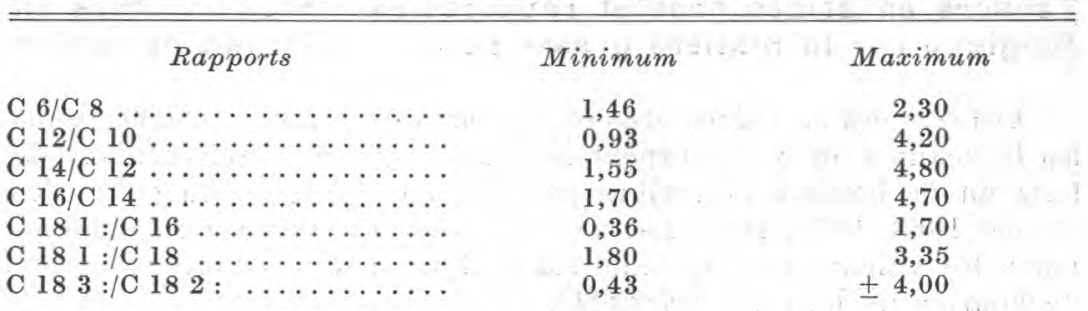

L'analyse chromatographique des esters méthyliques des acides gras en vue de la détermination d'une falsification éventuelle du beurre requiert toujours la connaissance de plusieurs éléments tels que les taux et certains rapports des acides gras. Dans les cas douteux, il est du plus haut intérêt de connaître l'origine de la matière grasse butyrique, et la plus grande prudence sera de rigueur avant de pouvoir émettre le moindre jugement. 


\section{Résumé}

La chromatographie gaz-liquide des esters méthyliques des acides gras a permis une étude poussée de la matière grasse butyrique de laits ou de beurres prélevés en Belgique.

Les variations de la composition en acides gras ont été étudiées suivant divers facteurs. Les valeurs extrêmes ont été observées et réunies en tableaux. De nombreux exemples et graphiques illustrent cette étude.

\section{Summary}

Gas-liquid chromatography of fatty acid methyl esters allowed to carry on a sharp study of milk and butter fat samples from Belgium.

The variations of fatty acid composition were studied in various respects. The extreme values were observed and collected on tables. Many examples and figures explain the results of this research.

\section{BIBLIOGRAPHIE}

[1] A.-L. Guyot et E.-F. Piraux. Etude par la chromatographie en phase gazeuse de la matière grasse des beurres de laiterie belges. Premières applications à la recherche des falsifications. Bull. Inst. agron. et Stat. Rech. Gembloux, 1964, 32, 2, 190.

[2] A.-L. Gurot. Composition de la matière grasse des beurres belges et recherche des falsifications par la chromatographie en phase gazeuse. Symposium III de chromatographie. Société belge des sciences pharmaceutiques. 1964.

[3] A.-L. Guyot et E.-F. Piraux. Chromatographie en phase gaz-liquide des principales graisses et huiles alimentaires. Remarques générales concernant la falsification des beurres à l'aide de plusieurs de ces graisses. Bull. Inst. agron. et Stat. Rech. Gembloux, 1965, 33, 1, 79.

[4] S. Kuzdzal et C. PaQuot. Exposés et communic. III. Sur l'identification d'un beurre de constitution anormale. Ann. Fals. Exp. Chim., 1962, ne 637 . 